medial orbitofrontal cortex to dorsal thalamus, amygdala and habenula nucleus. amSTN DBS improved cognitive flexibility, whereas VC/VS DBS did not, and affected a circuit connecting the amSTN with lateral orbitofrontal cortex, dorsolateral prefrontal cortex and dorsal anterior cingulate cortex. Both circuits have different functions and are known to be abnormal in OCD. The implications of these findings for the future management of severe OCD will be discussed.

*A Randomised Trial Directly Comparing Ventral Capsule and Anteromedial Subthalamic Nucleus Stimulation in Obsessive Compulsive Disorder: Clinical and Imaging Evidence for Dissociable Effects. Himanshu Tyagi, Annemieke M. Apergis-Schoute, Harith Akram, Tom Foltynie, Patricia Limousin, Lynne M. Drummond, Naomi A. Fineberg, Keith Matthews, Marjan Jahanshahi, Trevor W. Robbins, Barbara J. Sahakian, Ludvic Zrinzo, Marwan Hariz, and Eileen M. Joyce, Biological Psychiatry, in press.

\section{MENTAL IMAGERY AND MENTAL HEALTH SCIENCE: FROM LAB TO HOSPITAL TO A REFUGEE CAMP?}

Emily A Holmes. Uppsala University, Sweden

\subsection{6/jnnp-2019-BNPA.6}

Emily Holmes, PhD, DClinPsych is a Professor in Psychology at the Department of Psychology, Uppsala University, Sweden. She is also affiliated to the Karolinska Institutet's Department of Clinical Neuroscience, and is a Visiting Professor of Clinical Psychology at the Department of Psychiatry, University of Oxford, UK. Holmes received her degree in Experimental Psychology at the University of Oxford, UK. She is also a clinician and completed a clinical psychology training doctorate at Royal Holloway University of London, and a $\mathrm{PhD}$ in Cognitive Neuroscience in Cambridge, UK. Holmes' work as a clinical psychologist has fuelled her research questions. She is interested in psychological treatment innovation in mental health - both in creating new techniques and reaching more people. Under the wider umbrella of mental health science, her approach brings together psychology, neuroscience, psychiatry, maths and more. Holmes' research has demonstrated that mental imagery has a more powerful impact on emotion than its verbal counterpart. Her group is particularly interested in understanding and reducing intrusive memories after trauma. This is relevant for people after a traumatic event, whether a severe motor vehicle accident, traumatic childbirth or war. She is an Associate Editor for Behaviour Research and Therapy. She is on the Board of Trustees of the research charity 'MQ; transforming mental health'.

Traumatic events can affect anyone from a road traffic accident, to traumatic childbirth or war-related trauma for refugees. A core clinical symptom for many people who experience trauma comprises intrusive memories to the event. Intrusive memories that 'flash backwards' to past trauma occur in post-traumatic stress disorder (PTSD). They take the form of mental imagery, that is, an experience like perception in the absence of a percept, such as 'seeing in our mind's eye'. Indeed, intrusive, affect-laden mental images can cause distress across mental disorders. Mental images allow us to time travel, and can also 'flash forwards' to the future such as those can occur related to suicide or in bipolar disorder.

My clinical research group has an interest in understanding and treating maladaptive mental imagery via psychological therapies. To do this, we are curious about what we can learn from cognitive psychology and neuroscience to inform novel treatment development. I will discuss recent work concerning intrusive memory encoding, and a mechanistically informed intervention aiming to disrupt memory re-consolidation via dual task interference, thereby to reduce the frequency of intrusive memories. Recent work on tackling mood instability via focused imagery techniques will be discussed. A broader vision for science-informed psychological treatment innovation will also be explored from working hospitals (Iyadurai et al, 2018, Molecular Psychiatry) to further afield with refugees (Holmes, Ghaderi et al, 2018, Lancet Psychiatry).

\section{HOW NEUROSCIENCE CAN INFORM PUBLIC POLICY}

Facundo Manes. Professor of Behavioural Neurology and Cognitive Neuroscience INECO Foundation for Research in Cognitive Neuroscience and Institute of Neuroscience - Favaloro University. Argentina

\subsection{6/jnnp-2019-BNPA.7}

Facundo Manes is an Argentinian neuroscientist. He was born in 1969, and spent his childhood and adolescence in Salto, Buenos Aires Province. He studied at the Faculty of Medicine, University of Buenos Aires, where he graduated in 1992, and then at the University of Cambridge, England (Master in Sciences). After completing his postgraduate training abroad (USA and England) he returned to the country with the firm commitment to develop local resources to improve clinical standards and research in cognitive neuroscience and neuropsychiatry. He created and currently directs INECO (Institute of Cognitive Neurology) and the Institute of Neurosciences, Favaloro Foundation in Buenos Aires City. Both institutions are world leaders in original scientific publications in cognitive neuroscience. He is also President of the World Federation of Neurology Research Group on Aphasia and Cognitive Disorders (RGACD) and of the Latin American Division of the Society for Social Neuroscience. Facundo Manes has taught at the University of Buenos Aires and the Universidad Católica Argentina. He is currently Professor of Neurology and Cognitive Neuroscience, Faculty of Medicine and Psychology of the Favaloro University and was appointed Professor of Experimental Psychology at the University of South Carolina, USA. He has published over 100 scientific papers in the most prestigious original specialised international journals such as Brain and Nature Neuroscience. He has also given lectures at several international scientific fora as the 'Royal Society of Medicine' (London) and the 'New York Academy of Sciences', among others. His current area of research is the neurobiology of mental processes. He believes in the importance of scientific disclosure for Society. He led the program 'The Brain Enigmas' on Argentina TV and wrote many scientific articles in the national press. Finally, Prof. Facundo Manes is convinced that the wealth of a country is measured by the value of human capital, education, science and technology, and that there is the basis for social development.

In the last two decades, mainly in the developed world, we have seen a remarkable shift in how policies are made. Until the late 1990's, the ranks of lead policymakers comprised of economists, lawyers, and financial experts. Behavioral scientists are now increasingly being asked to bring their insights and expertise from the laboratory into the 'real' world. As a result, many experts in behavioral sciences are playing a much 